\title{
Wearing same- and opposite-sex virtual bodies and seeing them caressed in intimate areas
}

\author{
Manuel Mello ${ }^{\mathrm{a}, \mathrm{b},{ }^{*},}$ Martina Fusaro ${ }^{\mathrm{a}, \mathrm{b}}$, Gaetano Tieria,c, Salvatore Maria Agliotia,b, ${ }^{*}$ \\ a SCNLab, Fondazione Santa Lucia, IRCCS, Rome, Italy \\ b Sapienza, Università degli Studi di Roma \& CLNS@Sapienza, Istituto Italiano di Tecnologia, \\ Rome, Italy \\ c Virtual Reality Lab, University of Rome Unitelma Sapienza, Rome, Italy \\ * Manuel Mello; Salvatore Maria Aglioti \\ E-mail: manuel.mello@uniroma1.it; salvatoremaria.aglioti@uniroma1.it;
}

\section{Keywords}

Body ownership, Social touch, Virtual reality, Embodiment, Body swap, Sex differences

\section{Author Contributions}

M. M., M. F., G. T., and S. M. A.: Conceptualization, Methodology; M. M.: Investigation, Formal Analysis, Data Curation, Writing - Original Draft, Visualization; G. T.: Software; S. M. A.: Funding Acquisition; M. M., M. F., G. T., and S. M. A.: Writing - Review \& Editing.

\begin{abstract}
Immersive virtual reality enables people to undergo the experience of owning an artificial body and vicariously feeling tactile stimuli delivered to it. However, it is currently unknown how such experiences are modified by the sexual congruency between the human and the artificial agent. In two studies, heterosexual men (Experiment 1) and women (Experiment 2) embodied same-sex and opposite-sex avatars and were asked to evaluate the experience (e.g., pleasantness, erogeneity) of being touched on social or intimate areas of their virtual body by a male or female avatar. Electrocardiogram and galvanic skin response were also recorded. Moreover, participants' implicit and explicit gender biases were examined via a gender-potency implicit association test and the Ambivalent Sexism Inventory. When embodying a same-sex avatar, men and women rated caresses on intimate areas from an avatar of the opposite sex as more pleasant and erogenous. Conversely, body swap-i.e., wearing an opposite-sex avatar-enhanced participants' perceptions of pleasantness and erogeneity for caresses on intimate areas from a same-sex toucher. This effect was stronger in men than in women. Furthermore, physiological correlates of enhanced processing of arousing stimuli predicted behavioral outcomes during the body swap illusion. Wearing an opposite-sex avatar affects one's own body representations and may have important implications on people's attitudes and implicit reactivity to touch-mediated interactions. Men seem more susceptible to this type of body swap illusion. Our paradigm may induce profound changes of crosssex perspective-taking and provide novel tools for promoting empathy and comprehension of sexrelated diversity.
\end{abstract}

\section{Main Text}

\section{Introduction}

The ensemble of feelings, representations, and beliefs concerning the notion that the body and its parts belong to the "self" is referred to as body ownership (BO), an essential pillar of corporeal awareness (Berlucchi \& Aglioti, 2010). Intuitively, we think of BO as a stable mental construct. 
However, fast and profound BO changes are observed in brain-damaged patients who deny that their contralesional limb belongs to them (Vallar \& Ronchi, 2009; Moro et al., 2016; Blanke et al., 2004), as well as in healthy people who report the illusion of owning a physical (e.g., a rubber hand) or virtual limb as a consequence of synchronous visuo-tactile stimuli of the artificial and real body (Botvinick \& Cohen, 1998; Sanchez-Vives et al., 2010; Kilteni et al., 2012; Pyasik et al. 2020). Immersive virtual reality (IVR) has proven to be an invaluable tool for exploiting full-body illusion paradigms, wherein a person's real body is replaced with a virtual one and an illusory feeling of BO over the virtual body (VB) is created (the body swap illusion; Kilteni et al., 2015; Petkova \& Ehrsson, 2008). Studies indicate that mere observation of a VB from a first-person perspective (1PP; i.e., aligned with and spatially matching one's own real body) is sufficient to induce illusory BO (Slater et al., 2010; Pavone et al., 2016; Fusco et al., 2020; Monti et al., 2020; Keenaghan et al., 2020). Recent IVR studies have shown that illusory BO can affect people's perceptions and behavior as well as their implicit attitudes, depending on conspicuous features of the VB (Maister et al., 2015) such as ethnicity (Peck et al., 2013), age (Banakou et al., 2013), shape (Van Der Hoort et al., 2011), size (Preston \& Ehrsson, 2014; Provenzano et al., 2020), and sex (Slater et al., 2010; Peck et al., 2020).

It is relevant to the present study that even passive observation of painful or pleasant stimuli delivered to one's own VB may trigger vicarious sensations congruent with the observed stimuli (Fusaro et al., 2016, 2019, 2021). Fusaro and colleagues (2016, 2019), for example, reported that the observation of a virtual caress on an embodied virtual hand induced subjective feeling of vicarious touch, which were accompanied by changes in physiological reactivity (i.e., heart rate and skin conductance). The affective value of social touches such as a caress ranges from extreme pleasantness (e.g., erotic or consolatory feelings) to extreme unpleasantness (e.g., pain, disgust). Moreover, the age, ethnicity, and gender of both the toucher and receiver-as well as factors related to the context of the tactile stimulation-have all been found to affect how touch is perceived (Gallace \& Spence, 2010; Morrison et al., 2010). Tellingly, a recent extension of "virtual touch" studies (Fusaro et al., 2016, 2019) highlighted different patterns of behavioral and physiological responses to virtual caresses in heterosexual and homosexual participants, supporting that gender and sexual orientation (SO) play an important role in touch-mediated interactions (Fusaro et al., 2021).

What remains unknown is whether the pattern of behavioral and physiological reactivity to a samesex vs. opposite-sex toucher is different based on whether one's own VB is or is not coherent with one's own sex. The topic of sex-related body swaps has recently gained momentum (Slater \& Sanchez-Vives, 2014) after several virtual reality studies demonstrated that wearing opposite-sex VBs may reduce gender biases and/or enhance empathy and perspective-taking abilities (Peck et al., 2018; Seinfeld et al., 2018; Neyret et al., 2020; de Borst et al., 2020; Tacikowski et al., 2020). Here, in two different IVR experiments, heterosexual men (Experiment 1) and women (Experiment 2) embodied same-sex or opposite-sex avatars (i.e., swapping sexual appearance) and then observed virtual caresses delivered by a male or female avatar on different parts of their VB (Fig. 1; Movie S1). These body regions were defined as neutral (e.g., knee), social (e.g., hand), or intimate (e.g., pelvis) on the basis of an ad hoc survey reported in Fusaro and colleagues' study (21). Behavioral ratings were collected for each observed caress using visual analogue scales (VASs; Table 1). Physiological reactivity was recorded throughout the experiments in the form of galvanic skin response (GSR) and electrocardiogram (ECG). We predicted that embodying an opposite-sex VB would change heterosexual people's feelings and physiological reactivity by shifting them towards what was expected for their embodied VB. Specifically, if the body swap illusion works as predicted when heterosexual people embody an opposite-sex VB, we would expect increased feelings of pleasantness and erogeneity in response to same-sex intimate touch (i.e., men being virtually caressed by a male avatar and women being virtually caressed by a female 
avatar) when wearing an opposite-sex VB. Moreover, significant differences in physiological reactivity depending on the type of embodied VB are expected.

\section{Materials and Methods}

Participants. A total of 21 healthy heterosexual men (average age $=26.53, S D=3.92$; range $18-$ 36; Experiment 1) and 21 healthy heterosexual women (average age $=26.53$, $S D=3.92$; range 18-36; Experiment 2) participated in the study. Prior to being recruited for the experiment, participants rated their SO via a 0-to-100 Kinsey scale (Kinsey et al., 2003) ranging from "exclusively heterosexual" to "exclusively homosexual", with "bisexual" in the middle of the VAS scale (mean $\pm S D$ for male sample $=6.76 \pm 8.91$; mean \pm SD for female sample $=8.62 \pm 8.25$ ). We divided the VAS range into the Kinsey scale's 7 categories: $0-14.28=$ exclusively heterosexual; 14.28-28.57 = predominantly heterosexual, only incidentally homosexual; $28.57-42.85=$ predominantly heterosexual, but more than incidentally homosexual; $42.85-57.14$ = equally heterosexual and homosexual; $57.14-71.42=$ predominantly homosexual but more than incidentally heterosexual; $71.42-85.71=$ predominantly homosexual, only incidentally heterosexual; $85.71-100=$ exclusively homosexual. The cutoff to be included in our sample was 28.57. The sample size was chosen based on our previous work (Fusaro et al., 2021), where sample size estimations were performed using MorePower 6.0 software. The experimental protocol was approved by the ethics committee of the IRCCS Santa Lucia Foundation and followed the ethical standards of the 2013 Declaration of Helsinki. All participants gave their written informed consent to take part in the study and were naïve to the purposes of the research.

General procedures. All participants laid down on a beach chair placed in the laboratory room and wore a head-mounted display (HMD) through which they observed from 1PP a VB in underwear that substituted for their own body (see SI Appendix for a more detailed description of experimental stimuli and setup). In two different sessions, separated by a few days, participants embodied either a same-sex or an opposite-sex VB by means of a passive observation approach in 1PP, which has been described as a sufficient and necessary condition for inducing BO over a virtual avatar (Fig. 1; Movie S1; Slater et al., 2010; Pavone et al., 2016; Fusco et al., 2020; Monti et al., 2020; Keenaghan et al., 2020). Embodiment type order was randomized across participants. Each session consisted of two blocks during which participants observed virtual caresses delivered by either a female or male avatar. Thus, no actual touch was delivered to the participants. This is an important detail because the embodiment achieved by mere vision of a mannequin body is reduced with respect to a condition in which touch to participants' own, unseen body is added (Carey et al, 2019). The body parts touched by the avatar included the foot, knee, pelvis, chest, head, and hand. Four trials per body area were included in each block, with two delivered by the other avatar standing on the right side of the VB and the remainder two delivered by the other avatar standing on the left side of the VB. Thus, one block consisted of 24 touches in total. The six body areas were categorized as "neutral" (foot and knee), "social" (head and hand), or "intimate" (chest and pelvis). This categorization was based on a survey previously run by our research group (Fusaro et al., 2021). During the first minute, participants merely observed their VB aligned with their physical body. The experimental design included three experimental factors: embodiment type (whether the VB belonged to the same or the opposite sex), the sex of the touching avatar (male or female), and body area (neutral, social, or intimate). 

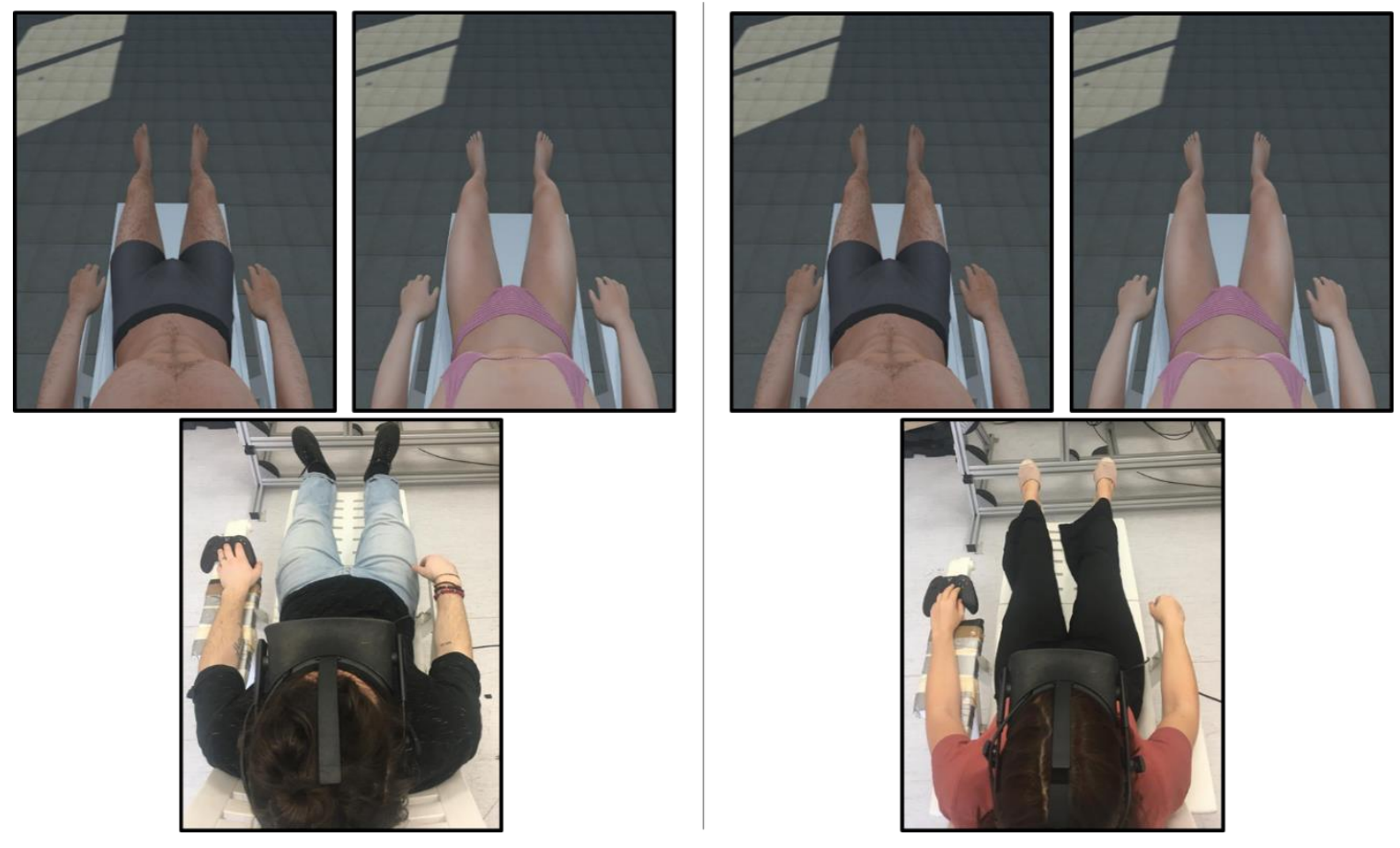

Figure 1. Participants laid down on a beach chair and, through an HMD, saw from a first-person perspective a VB in underwear that replaced their own body. Left panel: depending on the experimental session, men embodied either a male (same-sex) or a female (opposite-sex) VB. Right panel: depending on the experimental session, women embodied either a male (opposite-sex) or a female (same-sex) VB.

Each caress lasted approximately $3 \mathrm{~s}$ and had a velocity of approximately $3 \mathrm{~cm} / \mathrm{s}$, regarded as the main feature of pleasant affective touch (Löken et al., 2009). After each caress was delivered, participants kept observing their own VB for $7000 \pm 500 \mathrm{~ms}$. At the end of the trial, they were presented with four VASs through which they provided ratings (moving a joystick-controlled cursor) about their touch-evoked experiences (Table 1). Moreover, at the end of each block, participants were asked to rate their feelings of virtual embodiment (ownership, identification, comfortableness) over the VB as well as vicarious touch in response to different VAS statements and questions (Table 1). For both in-session and end-of-block VASs, scores ranged from 0 to 100, where 0 meant absence of the feeling (e.g., no ownership, not pleasant, not erogenous) and 100 corresponded to a very strong feeling. At the end of each session (i.e., after the main experimental task), participants were asked to complete a gender-potency implicit association test (IAT, Greenwald et al., 1998; Rudman et al., 2001) and an online version of the ambivalent sexism inventory (ASI, Glick \& Fiske, 1996; Italian validation: Manganelli Rattazzi et al., 2008; for further details on IAT and ASI analyses and results, see SI Appendix, Additional results.

Table 1: In-session and end-of-block VAS questions and statements. Scores ranged from 0 to 100 , where 0 meant that the caress was not appropriate, not pleasant, not arousing, and not erogenous. A score of 100 meant that the caress was extremely appropriate, pleasant, arousing, and erogenous. As for end-of-block questions and statements, a score of 0 meant the absence of feelings of ownership, vicarious touch, identification, or comfortableness with the virtual body seen in 1PP, while a score of 100 meant corresponding very strong feelings. 


\begin{tabular}{|l|l|}
\hline Category & Question/Statement \\
\hline Appropriateness & How appropriate was the touch? \\
\hline Arousal & How arousing was the touch? \\
\hline Pleasantness & How pleasant was the touch? \\
\hline Erogeneity & How erogenous was the touch? \\
\hline Ownership & $\begin{array}{l}\text { "It seemed like I was watching my body" } \\
\text { "It seemed like the virtual body was my body" }\end{array}$ \\
\hline (Ownership) Control & $\begin{array}{l}\text { "It seemed like I had more than one body" } \\
\text { "It seemed like I did not have my body anymore" }\end{array}$ \\
\hline Vicarious touch & "It seemed like I was feeling the touches on my body" \\
\hline Comfortableness & "How comfortable did you feel in the virtual body?" \\
\hline Identification & "To what extent did you identify yourself with the body \\
you observed in 1PP?"
\end{tabular}

Physiological recordings and pre-processing. SCR and ECG were used as measures of physiological reactivity to virtual social and intimate touch. An ADInstruments PowerLab 8/35 device was used as a signal amplifier along with the ML116 GSR Amplifier (providing a $75 \mathrm{~Hz}$ AC excitation with low constant voltage of $22 \mathrm{mVrms}$ ) with specific GSR sensors consisting of two bipolar finger electrodes. The sensors were applied on the distal phalanx of the index and middle fingers of the right hand, and the signal was sampled at $1 \mathrm{KHz}$. For the ECG, two electrodes (DORMO pre-gelled electrodes, $50 \mathrm{~mm}$ ) were placed on the back of each hand, and the reference was placed on the left ankle. Signals were sampled at $1 \mathrm{kHz}$ and filtered using a $30 \mathrm{~Hz}$ low-pass filter. Data were recorded using the LabChart 7 software (ADInstruments, Inc.).

Inter-beat intervals were computed and then converted into heart rate $(\mathrm{HR})$ in beats per minute (bpm) using LabChart. Data were reduced offline in $1 \mathrm{~s}$ bins. HR changes contingent upon observation of virtual caresses were computed as differential values between $3 \mathrm{~s}$ after a virtual caress was delivered and $2 \mathrm{~s}$ of baseline before the appearance of the touching avatar. Raw skin conductance data were extracted from LabChart as .txt files and entered in MATLAB for analysis. The Ledalab toolbox was used to run a discrete decomposition analysis through which we separated phasic from tonic activity. The pre-processing steps included down-sampling, smoothing, and Butterworth filtering of the raw data. Event-related SCR was averaged across $6 \mathrm{~s}$ after a virtual caress was delivered (Fusaro et al., 2016) and transformed using square root transformation. Responses below $0.1 \mu \mathrm{S}$ were discarded.

Data analysis. We performed a linear mixed-effects analysis using the scores for each of the four in-session VAS questions and end-of-block statements (ownership, comfortableness, identification, and vicarious touch) as outcome and the interactions among all our experimental factors (embodiment type, touching avatar, and body area) as predictors. Moreover, linear mixed-effects analyses were run with baselined HR in bpm and SCR in $\mu$ S as outcome. The fitted models included by-subject intercepts, as well as by-subject slopes for the effects of embodiment type, touching avatar, and body area (when doing so did not produce model overfitting, in which case model complexity was reduced). For the in-session VAS questions and physiological measures, nonorthogonal planned comparisons for the body area factor were performed. Specifically, the social and intimate levels were both compared separately to the neutral level. When relevant, specific comparisons between conditions were examined using post hoc tests (Bonferroni-corrected, or Tukey-corrected for trend analyses). Statistical modelling was carried out in $\mathrm{R}$ using the function Imer() from the Ime4 package (Bates et al., 2007). Model complexity was gradually increased by inserting fixed effects and their interactions to check for the model that best fitted the data. The different models were compared using the anova() function from the stats package in $R$ ( $R$ Core Team, 2019). AIC, BIC, and Chi-square statistics informed us on which model best fitted the data compared to the previous ones in the hierarchy. Graphical inspection of model residuals and fitted 
vs. predicted values revealed that normality of model residuals, homoscedasticity and linearity assumptions were met for all the statistical models. Post hoc tests were performed using the Ismeans() function from the Ismeans package (Lenth, 2017). Trend analyses on physiological measures were performed using the emtrends() function from the emmeans() package (Lenth, 2020). Model effect sizes were computed using the r.squaredGLMM() function from the MuMln package (Barton, 2020).

\section{Results}

\section{Experiment 1. Only heterosexual male participants}

Feelings of embodiment and vicarious touch. Participants' virtual embodiment was evaluated through a series of questions and statements related to their feelings of ownership, identification, and comfortableness regarding the VBs. One statement specifically assessed vicarious feelings for virtual touches (see Methods and Table 1). We found a significant effect of embodiment type (same-sex versus opposite-sex VB) on ownership ratings (estimate $=6.91 ; t$-value $=-2.3, p=0.02$; $R^{2}$ conditional $\left.=0.66\right)$. This was explained by the higher ownership ratings when embodying a samesex male VB $(59.15 \pm 4.66)$ compared to an opposite-sex female VB $(52.23 \pm 4.66)$. No other main effects or interactions were significant. Additionally, men identified with a male VB more than a female VB (estimate $=-14.02 ; t$-value $=-2.26, p=0.04 ; R^{2}$ conditional $\left.=0.38\right)$ (Fig. $2 \mathrm{~A}$ ), while feeling equally comfortable in both conditions (estimate $=-3.13$; $t$-value $=-0.87, p=0.39$ ). Female avatar touch was found to generate stronger vicarious tactile feelings $(M=48.28, S E=4.51)$ than male avatar touch $(M=40.94, S E=5.27)$.

Pleasantness of touch. Overall, participants (all men) rated female avatar touch as more pleasant $(M=49.35, S E=2.93)$ than male avatar touch $(M=37.14, S E=2.69$; estimate $=-12.93 ; t$ value $=-4.92, p<0.001$ ). The embodiment of participants (all men) in a female VB produced a significant increase in the reported pleasantness of caresses on intimate areas from a male avatar (estimate $=13.97 ; t$-value $=4.89 ; p<0.001 ; R^{2}$ conditional $=0.60 ;$ Fig. $2 \mathrm{~B}$ ). Direct comparison between specific conditions (Bonferroni-corrected) revealed that pleasantness ratings for intimate touch from a male avatar differed significantly between the same-sex and opposite-sex conditions (estimate $=-12.16$; $t$-ratio $=-4.56, p=0.003$ ). This comparison specifically informs us of the degree of change in attitudes that depends on embodying an opposite-sex VB. Specifically, caresses on intimate areas from a male avatar were rated as more pleasant during opposite-sex embodiment (wearing a female VB; $40.16 \pm 3.71$ ) than during same-sex embodiment (wearing a male VB; $28 \pm 3.49)$

Erogeneity of touch. Intimate touch was rated as more erogenous $(M=38.63, S E=4.13)$ than neutral touch $(M=25, S E=3.18)$ (estimate $=15.58 ; t$-value $=4.55 ; p<0.001)$. The embodiment of participants (all men) in a female VB produced a significant increase in the erogeneity of caresses on intimate areas from a male avatar compared to neutral touch (estimate $=16.33$; $t$-value $=4.78$; $p<0.001, R^{2}$ conditional $=0.68$; Fig. $2 \mathrm{~B}$ ). No such difference was found when participants embodied a male VB. As with pleasantness ratings, post hoc tests showed that erogeneity ratings for intimate touch from a male avatar differed significantly between the same-sex and opposite-sex conditions (estimate $=-28.72, S E=3.29 ; t$-ratio $=-8.74, p<0.001$ ). Specifically, erogeneity for caresses on intimate areas delivered by a male avatar was rated $11.6 \pm 4.17$ during same-sex embodiment, while it was rated $40.32 \pm 4$ during opposite-sex embodiment.

Correlation analyses. The results presented above suggest, as expected, that the levels of pleasantness and erogeneity that heterosexual men experience for vicarious touch depend on the VB they are embodying. To provide additional evidence supporting the relationship between embodying a VB and vicarious touch sensation, we ran correlation analyses between the ownership scores and VAS ratings of pleasantness and erogeneity. For each dimension, we computed an index wherein ratings of neutral caresses were subtracted from ratings for caresses on intimate 
and social areas (intimate minus neutral index, social minus neutral index). Bonferroni correction for multiple comparisons returned a $p$-value of 0.003 ( $p$-value of $0.05 / 16$, as hypotheses were tested on two different dimensions [pleasantness and erogeneity], two indexes, and four conditions, namely male toucher/same-sex embodiment, female toucher/same-sex embodiment, male toucher/opposite-sex embodiment, and female toucher/opposite-sex embodiment). We found two significant positive correlations between erogeneity ratings for caresses on intimate areas (intimate minus neutral index) and ownership scores. The first (Spearman' $\rho=0.46, p<0.001$ ) refers to the condition in which male participants embodying a male VB were caressed by a female avatar. Specifically, the more ownership that participants felt over the male VB, the greater their reported erogenous sensation for caresses on intimate areas from a female virtual avatar. The second correlation result (Spearman' $\rho=0.5, p<0.001$ ) suggests that the more ownership that male participants felt over the female VB (sex-related body swap), the greater their erogenous sensation for intimate same-sex touch (caresses delivered by male avatars; Fig. 2C).

A

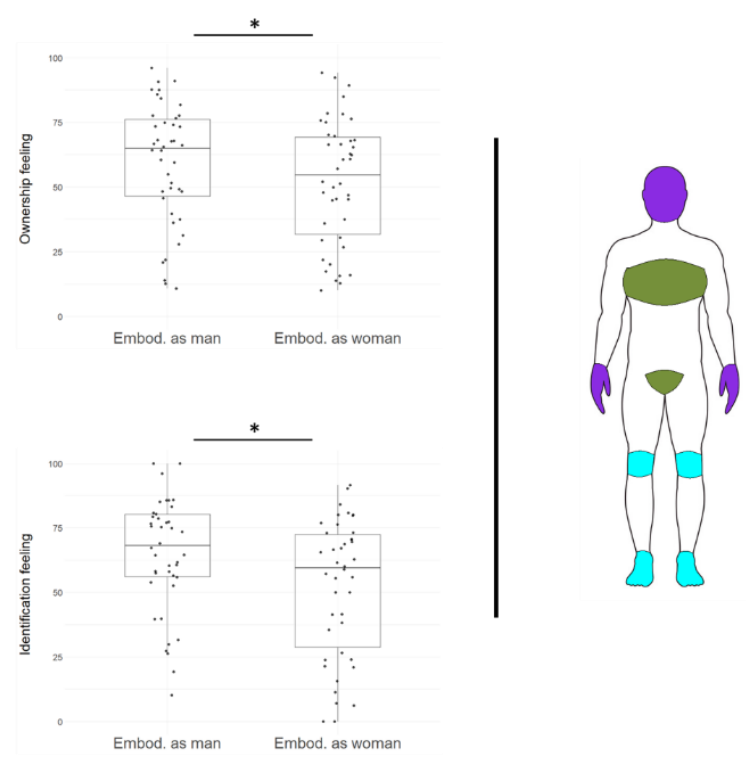

B

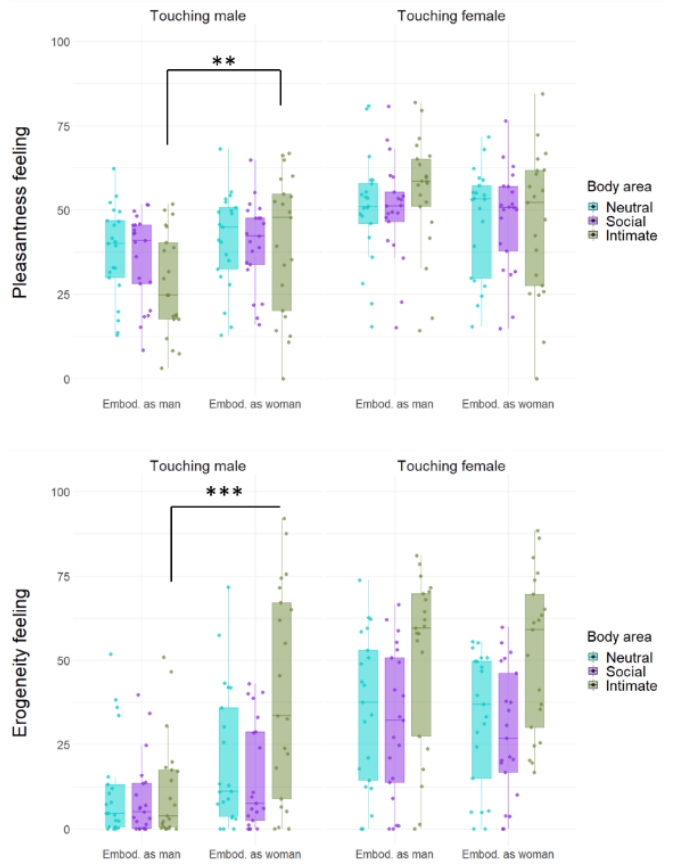

C

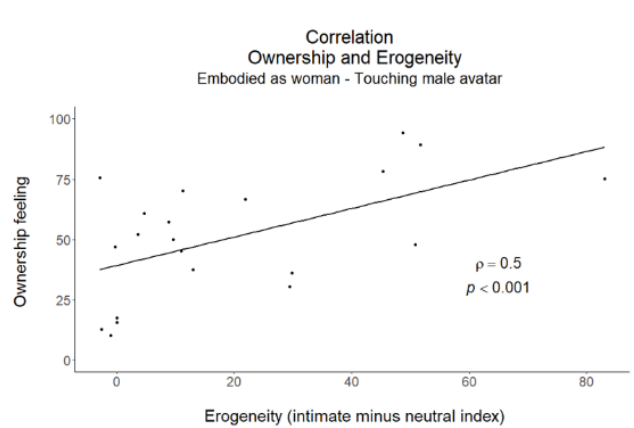

Figure 2. Main behavioral results for Experiment 1 (all men). (A): Box plots showing that men felt more ownership towards and identified more with a same-sex (male) than an opposite-sex (female) VB.(B) 
Pleasantness and erogeneity ratings provided by Experiment 1 participants (all men). (C) Scatter plots of correlation analysis (Spearman's rho) between ownership and erogeneity scores. The more ownership that men felt over the opposite-sex VB, the higher their ratings of erogeneity for same-sex intimate touch. Notes: Error bars represent mean SEs. Black lines indicate significant direct comparisons. * = significant at $<0.05$; ${ }^{* *}=$ significant at $<0.01 ;{ }^{* * *}=$ significant at $<0.001$.

Due to space limitations, the main findings related to the appropriateness and arousing power VASs are reported in the SI Appendix. This choice was motivated by the fact that appropriateness and arousal were less influenced by participants' embodiment in same-sex vs. opposite-sex avatars, which is related to our main hypothesis. The same is true for Experiment 2.

Physiological reactivity. Regarding the heart rate measurement, caresses on intimate body areas-independent of which avatar delivered them or whether participants embodied a male or female avatar-produced a heart rate deceleration (with respect to the baseline) compared to neutral caresses (estimate $=-0.75 ; t$-value $=-2.16, p=0.03 ; R^{2}$ conditional $=0.11$ ).

The analyses of skin conductance reactivity yielded a main effect of embodiment type (estimate $=-0.07 ; t$-value $=-2.89, p=0.003 ; R^{2}$ conditional $=0.31$ ). Participants' skin conductance response (SCR) was found to be higher in same-sex VB conditions $(M=0.82 \mu S, S E=0.08)$ compared to opposite-sex VB conditions $(M=0.75 \mu \mathrm{S}, S E=0.08)$. We also found a significant main effect of the touching avatar (estimate $=-0.05$; $t$-value $=-2.1, p=0.03$ ). More specifically, when heterosexual male participants were touched virtually by a female avatar, the average SCR was higher $(M=0.81 \mu S, S E=0.08)$ than when they were touched by a male avatar $(M=0.75 \mu S$, $\mathrm{SE}=0.08)$.

To investigate the possibility that participants' physiological activation influenced their behavioral ratings, different statistical models were fitted with baselined HR and SCR as predictors of both erogeneity and pleasantness scores.

Trend analyses on baselined HR revealed that higher erogeneity scores were predicted by lower HR when men were virtually caressed by a male avatar and embodied an opposite-sex VB, compared to a same-sex VB (Fig. $3 \mathrm{~A}$; estimate $=0.57$; $t$-ratio $=2.91, p=0.01$ ). Furthermore, trend analyses on SCR revealed that higher erogeneity scores were predicted by higher SCR when men were caressed by a male touching avatar and embodied an opposite-sex VB, compared to when they embodied a same-sex VB (Fig. 3B; estimate $=-4.10, S E=0.71$; $t$-ratio $=-5.77, p<0.001$ ).

As concerns pleasantness ratings, we found that higher pleasantness scores were predicted by higher SCR when men were caressed in intimate areas by a male touching avatar and embodied 
an opposite-sex VB, compared to when they embodied a same-sex VB (Fig 3C; estimate $=-3.39$; $t$-ratio $=-3.89, p=0.005)$.

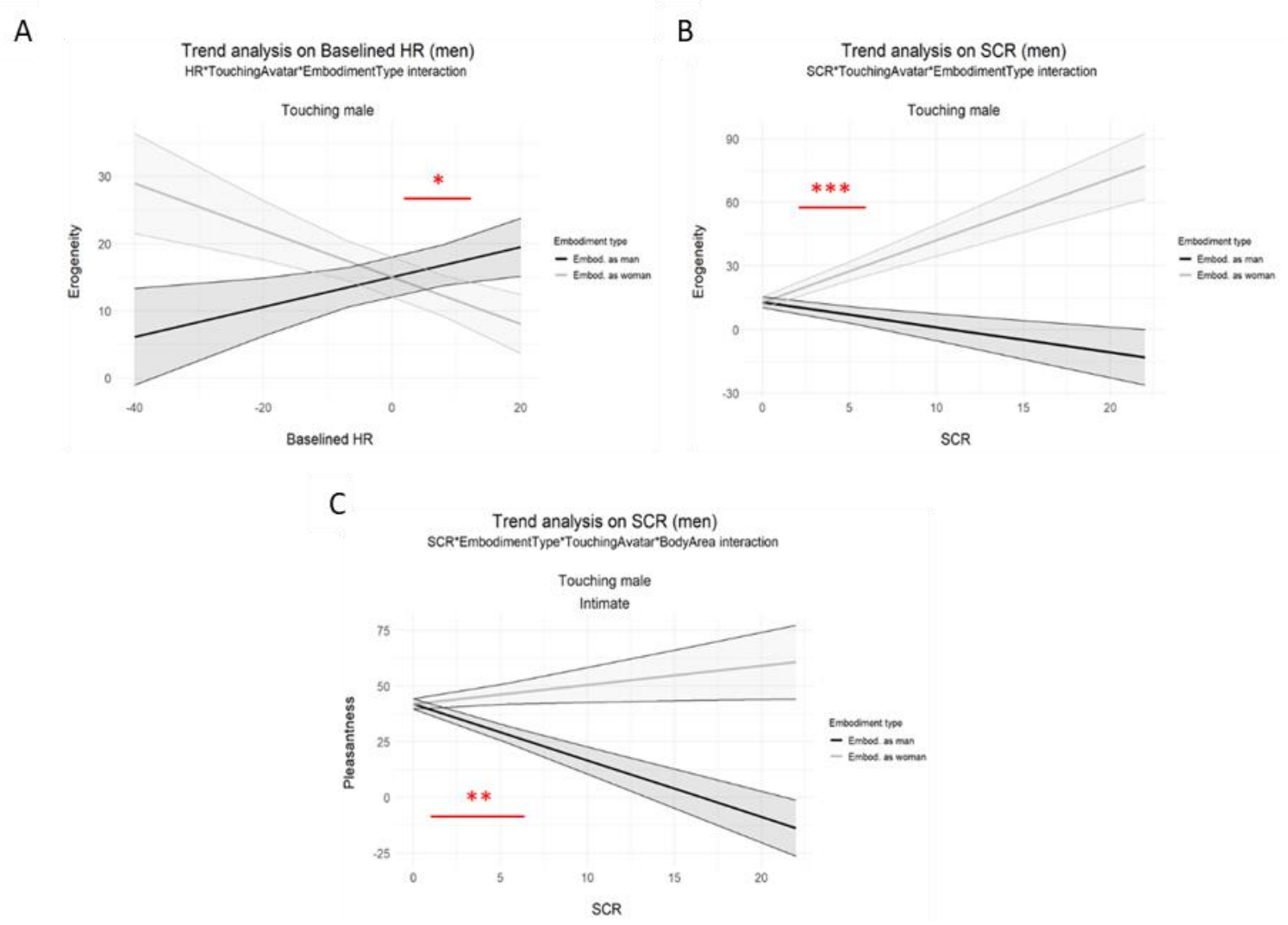

Figure 3: For erogeneity ratings as outcome, (A) HR trends and (B) SCR trends differed depending on the touching avatar's sex and the type of embodiment. (C): For pleasantness ratings as outcome, SCR trends differed depending on the touching avatar's sex, the type of embodiment, and the caressed body area. Notes: Error bars represent mean SEs. Red lines indicate significant post hoc comparisons. ${ }^{*}=$ significant at $<0.05$; ${ }^{* *}=$ significant at $<0.01 ;{ }^{* * *}=$ significant at $<0.001$.

Gender-potency IAT. We hypothesized that the implicit gender-potency bias would be reduced by embodying an opposite-sex VB. The strength of the association that sees men as powerful and women as weak (D1 score) was significantly different from zero in men (mean D1 $=0.38 ; t=7.43$, $p<0.001$ ). This means that men were quicker to associate male first names with power-related words and female first names with weakness-related words, compared to the opposite. IAT D1 scores did not differ across the two embodiment conditions (estimate $=0.02 ; \mathrm{t}=0.32, p=0.75$ ). Correlation analyses were run between IAT D scores (averaged across the two sessions) and two measures taken as indexes of behavior change in IVR, i.e., ownership scores during the oppositesex conditions and erogeneity scores for same-sex touch in intimate areas during opposite-sex conditions (intimate minus neutral index). We found no significant correlations between these measures.

ASI. Three participants did not complete this online questionnaire. An average ASI score for men was obtained by mediating across individual ASI scores. This score was compared with a sample normative mean from the Italian validation of the ASI questionnaire (ASI mean for men $=2.34$; Manganelli Rattazzi et al., 2008). A one-sample t test showed a significant difference between our 
sample mean and the normative mean taken as comparison ( $\mathrm{t}$ value $=-2.93, \mathrm{p}=0.009$ ). Specifically, men in our sample showed, on average, lower explicit ambivalent sexism (mean = 1.65 ) than did the normative sample (mean $=2.34$ ). Moreover, this process was repeated with the hostile sexism (HS) and benevolent sexism (BS) ASI subscales (Glick \& Fiske, 1996). Both HS and BS scores in our sample (HS mean $=1.76$; $B S$ mean $=1.53$ ) were significantly lower than the respective normative means (HS normative mean $=2.46$, $\mathrm{t}$ value $=-2.44, \mathrm{p}=0.02$; $\mathrm{BS}$ normative mean $=2.23$, $t$ value $=-2.62, p=0.01$ ). Correlation analyses were run between the $A S I$, as well as its HS and BS subscales, and the same ownership and erogeneity scores used with the IAT D1 values. We found no significant correlations between these measures.

\section{Experiment 2. Only heterosexual female participants}

Embodiment and vicarious touch feelings. We found a main effect of embodiment type on ownership scores When embodying a same-sex body, women rated their BO as higher-on average, $58.87 \pm 5.6$ compared to $36.55 \pm 5.6$ in opposite-sex conditions (estimate $=-22.33$; $t$ value $=-5.25, p<0.0001 ; R^{2}$ conditional $=0.83$ ). Additionally, women identified more with and felt more comfortable in the female VB than the male VB (Fig. 4A; identification: estimate $=-12.95, t$ value $=-3.25, p=0.001 ; R^{2}$ conditional $=0.62 ;$ comfortableness: estimate $=9.32, \quad t$-value $=-2.37$, $p=0.02 ; R^{2}$ conditional $=0.6$ ). We found no significant effects concerning feelings about vicarious touch in women, suggesting that women's vicarious sensations did not differ across conditions.

Pleasantness of touch. Women rated intimate touch as less pleasant $(M=34.75, S E=3.41)$ and social touch as more pleasant $(M=52.77, S E=2.76)$ than neutral touch $(M=40.94, S E=3.49)$. The interaction among embodiment type, touching avatar, and body area was significant for the contrast between intimate and neutral areas, with an estimate of -5.89 , a $t$-value of -1.99 , and probability $p$ of $0.04\left(R^{2}\right.$ conditional $=0.65$; Fig. $\left.4 \mathrm{~B}\right)$. As shown in Figure $4 \mathrm{~B}$, women rated caresses from a female avatar as equally pleasant compared to caresses from a male avatar during samesex conditions (i.e., while wearing a female VB). Moreover, no effect was found related to the caressed body area. Wearing a male VB (body swap of women to men) increased women's ratings of pleasantness for caresses from a female avatar on intimate areas. However, unlike in Experiment 1 (all men), Bonferroni-corrected post hoc analyses revealed that the ratings for caresses delivered by a female avatar on intimate areas did not differ significantly across the two embodiment conditions (estimate $=-9.68 ; t$-ratio $=-2.88, p=0.19$ ).

Erogeneity of touch. Overall, caresses from male avatars were rated as more erogenous $(M=26.22, S E=4.09)$ than those from female avatars $(M=24.28, S E=3.91)$. Compared to wearing a same-sex avatar, women's body swap (wearing a male VB) led to increased ratings of erogeneity for caresses from a female avatar on intimate areas and decreased ratings of erogeneity for caresses from a male avatar on intimate areas (estimate $=-9.4, t$-value $=-3.18, p=0.001$; $R^{2}$ conditional $=0.74$; Fig. 4B). However, direct comparisons between conditions did not show relevant differences. In particular, ratings of erogeneity for caresses on intimate areas delivered by a female avatar (estimate $=-10.24 ;$ ratio $=-3.35, p=0.07$ ) or a male avatar (estimate $=6.57 ; t$ ratio $=2.14$, 
$p=0.59$ ) did not significantly differ across embodiment conditions (same-sex vs. opposite-sex embodiment).
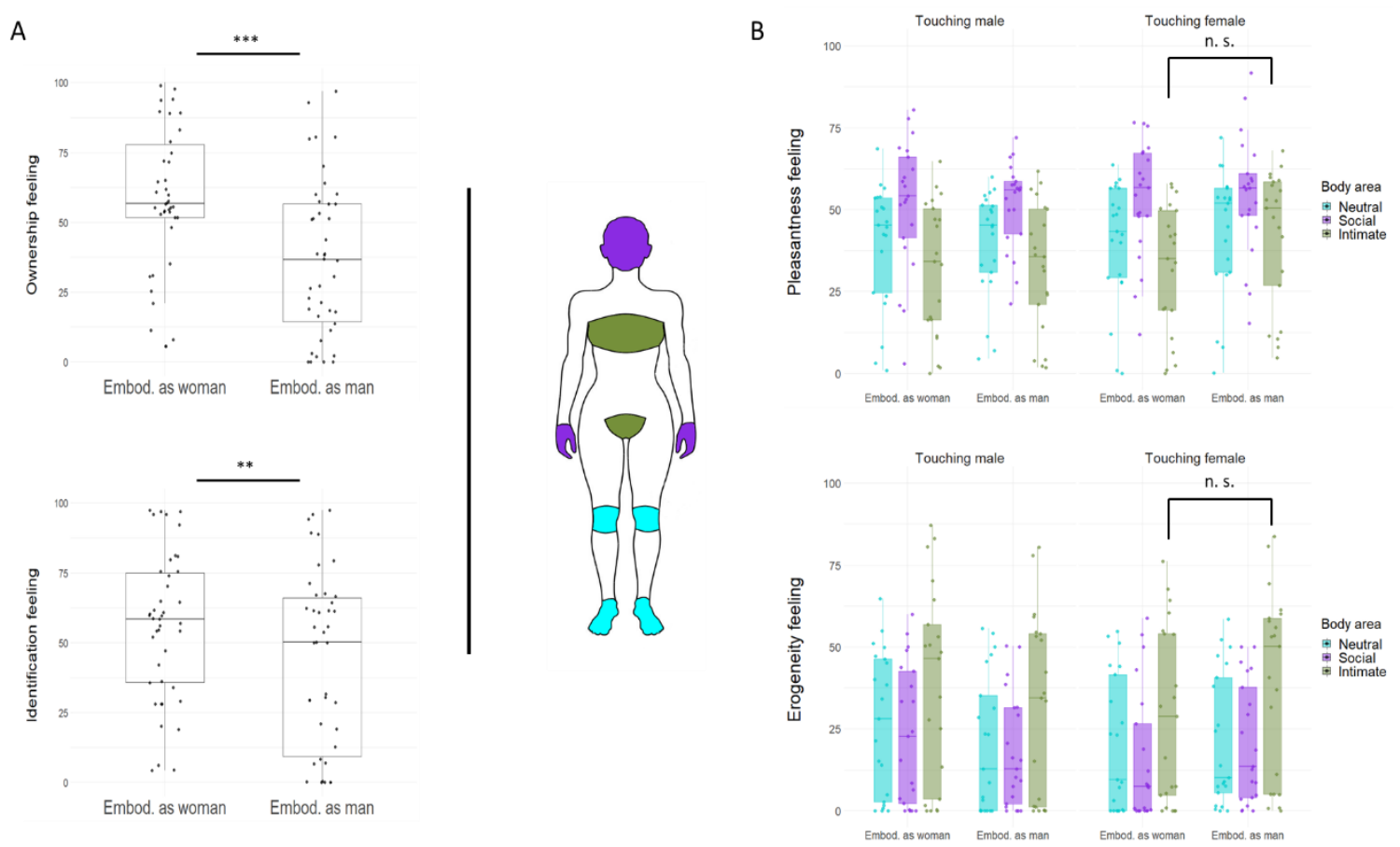

Figure 4: Main behavioral results for Experiment 2 (all women). (A): Box plots showing that women felt more ownership over and identified more with a same-sex (female) than an opposite-sex (male) VB. (B) Pleasantness and erogeneity ratings provided by Experiment 2 participants (all women). Notes: Error bars represent mean SEs. Black lines indicate significant (and nonsignificant) direct comparisons. ${ }^{* *}=$ significant at $<0.01 ;{ }^{* * *}=$ significant at $<0.001 ;$ n. $\mathrm{s} .=$ not significant.

Correlation analyses. The same criteria as those used in Experiment 1 were adopted for correlation analyses in Experiment 2. We found a significant negative correlation (Spearman' $\rho=-0.38, p=0.002$ ) between pleasantness scores for caresses on intimate areas and ownership scores. This correlation was specific to the condition in which women were caressed by a female avatar and embodied a same-sex VB; that is, female participants who reported higher ownership towards a female VB rated caresses on intimate areas by a female avatar as less pleasant.

Physiological reactivity. We did not find any significant effect with regard to the heart rate measure (see SI Appendix for means and standard errors for each experimental condition).

Concerning SCR, caresses delivered by female avatars elicited a higher SCR $(M=0.82, S E=0.1$ $\mu S)$ than caresses delivered by male avatars $(M=0.65, S E=0.1 \mu S)$. Moreover, caresses on intimate areas $(M=0.75, S E=0.1 \mu S)$ and social areas $(M=0.76, S E=0.1 \mu S)$ elicited a stronger SCR than caresses on neutral areas $(M=0.69, S E=0.1 \mu \mathrm{S})$. Finally, women's embodiment in a 
male VB (body swap) increased their SCR to female touching avatars (estimate $=-0.13$; $t$-value $=-2.56, p=0.01 ; R^{2}$ conditional $=0.41$ ).

As for experiment 1, statistical models were fitted with physiological measures as predictors of women's behavioral ratings. We found a significant interaction between SCR, embodiment type and touching avatar for erogeneity scores as outcome (estimate $=-3.17 ; t$-value $=-4.69, p<0.001$; $R^{2}$ conditional $\left.=0.74\right)$. Trend analyses revealed that higher erogeneity scores were predicted by higher SCR when women were caressed by a female touching avatar and embodied an opposite-sex VB, compared to when they embodied a same-sex VB (estimate $=-1.44 ; t$-ratio $=-3.30, p=0.005$ ).

IAT. One participant was excluded from these analyses for reasons relating to technical problems during the task. D1 scores in women were not significantly different from zero (mean D1 $=0.01 ; \mathrm{t}$ $=0.21, \mathrm{p}=0.83$ ). IAT $\mathrm{D}$ scores did not differ across the two embodiment conditions (estimate $=$ $0.07 ; t=0.9, p=0.38$ ). Same correlation analyses as in experiment 1 were run for women. We found no significant correlations between these measures.

ASI. One participant did not complete this online questionnaire. A one-sample t test performed on mean ASI score from our sample showed that women expressed lower explicit ambivalent sexism (mean $=1.61)$ than did the normative sample (mean $=2.08)$. Furthermore, HS and BS scores in our sample $(\mathrm{HS}$ mean $=1.45$; $\mathrm{BS}$ mean $=1.78$ ) tended to be lower than the respective normative means (HS normative mean $=1.90$, $\mathrm{t}$ value $=-1.97, p=0.06$, not significant; $\mathrm{BS}$ normative mean $=2.25$, $\mathrm{t}$ value $=-2.60, p=0.01$ ). Same correlation analyses as in experiment 1 were run for women. We found no significant correlations between these measures.

\section{Across-studies comparisons}

We decided to focus on separate experimental effects in men and women for several reasons. First, men and women were tested at two different times. Second, and more importantly, we conceived the study as a two-experiment one because we thought that focusing on men and women separately can better shed light on the effects of sex-related body swap on interpersonal touch preferences in both sexes. Third, we did not have any specific hypothesis concerning sex differences in sex-related body swap. In other words, we preferred parsimony over complexity, as the addition of a fourth factor in our experimental design would have made some results uninterpretable. . However, across-studies differences in behavioral and physiological reactivity to virtual caresses were analyzed through a between-subjects analysis, the results of which are described in detail in the SI Appendix. Here, we report the main findings. The embodiment of women in an opposite-sex body was found to decrease the illusion of being touched on their own body (vicarious touch VAS), both for caresses delivered by male and female avatars. In contrast, when men embodied a female body, caresses delivered by a male avatar produced a stronger illusion of being touched on their own body, compared to same-sex conditions (estimate $=18.74$; $t$-value $=2.13, p=0.03$ ). As outlined above, the significant direct comparison of different subjective ratings concerning same-sex intimate touch when wearing a same-sex compared to an oppositesex VB indicates that the consequences of the sex-related body swap illusion are stronger in men than in women. This was true for both pleasantness and erogeneity ratings. In line with this, we found that a between-subjects examination of these two VAS ratings yielded four-way interactions, which are explained by the stronger feelings of pleasantness (estimate $=19.85 ; t$-value $=4.83$, $p<0.001 ; \quad R^{2}$ conditional $=0.63$ ) and erogeneity (estimate $=25.72 ; \quad t$-value $=5.7, \quad p<0.001$; $R^{2}$ conditional $=0.71$ ) for same-sex intimate touch (compared to neutral) in men while embodying an opposite-sex VB. Furthermore, the difference in ownership ratings between same- and oppositesex conditions was stronger in women than in men (estimate $=15.48$; $t$-value $=3.52, p<0.001$; $R^{2}$ conditional $=0.72$ ), suggesting that men were more prone to embody an opposite-sex VB. $A$ between-subjects analysis was run on gender-potency IAT D scores and Ambivalent Sexism Inventory (ASI) scores. The difference between men and women in gender-potency stereotypes was statistically significant (estimate $=0.39 ; t=3.98, p<0.001$ ). Men had a stronger genderpotency bias than women (men: mean $\mathrm{D}$ score $\pm \mathrm{SD}=0.37 \pm 0.06$; women: mean $\mathrm{D}$ score $\pm \mathrm{SD}$ 
$=0.01 \pm 0.05$ ). No differences between men and women were found in explicit sexist attitudes (ASI: $t=-0.11, p=0.9$; HS: $t=-0.86, p=0.39$; BS: $t=0.78, p=0.44$ ). Finally, while higher SCR was found in women when they were caressed by a female touching avatar, compared to a male one, no such difference was observed in men (estimate $=0.11 ; t$-value $=3.14, p=0.001$ ).

\section{Discussion}

Wearing an opposite-sex virtual body. Capitalizing on the transformational power of IVR (Monti \& Aglioti, 2018), we explored the behavioral and physiological consequences of embodying a same-sex or an opposite-sex VB and observing it being caressed on different regions by male and female avatars. First, we found that, in both men and women, the feeling of BO over the VB was higher when its sex matched the participant's. Despite this, ratings of illusory BO for an oppositesex VB were not null, thus providing a frame in which to interpret the reactivity to intimate touch when embodying different types of VBs. Men appear to be more susceptible to the sex-related body swap illusion than women in terms of feelings of BO, comfortableness, and identification. Importantly, a comparison of men's and women's BO scores-reported in SI Appendix, Betweensubjects analysis, and Fig. S2-showed that women experienced significantly less ownership than men in the opposite-sex VB wearing condition.

Previous studies indicate that IVR users can experience faithful reproductions or replacements of their own body through which they can interact with virtual social environments. Bodily perception and representation can be easily manipulated by inducing in participants the feeling of "owning" a body different than the one they usually experience (i.e., their own body), as has been shown for avatars belonging to different demographic groups (Slater et al., 2010; Peck et al., 2013; Banakou et al., 2013; Peck et al., 2020; Tacikowski et al., 2020). The explicit and implicit attitudes and behavior of people undergoing this illusion of BO have been found to be affected by such a body swap in a way that is coherent with the new body they are experiencing (Maister et al., 2015; Slater \& Sanchez-Vives, 2014). Recent studies investigating sex-related body swaps are highly relevant for our purposes-i.e., the embodiment of men and women in opposite-sex VBs and its consequences for sex and gender biases and sex-related behaviors. In a pivotal example, Slater and colleagues (2010) reported that men could embody a female-appearing avatar and react behaviorally and physiologically to a threatening stimulation as if the VB were their own, provided that the embodiment happened in 1PP. More focused studies have recently examined the effects of sex-related body swaps on stereotype threat (Peck et al., 2018, 2020), working memory (Peck et al., 2020), gender violence and sexual harassment (Seinfeld et al., 2018; Neyret et al., 2020; de Borst et al., 2020), and gender identity (Tacikowski et al., 2020). Tacikowski and colleagues (2020) recently demonstrated that successful (and strong) sex-related body swap illusions are associated, in both women and men, with an online update of gender identity aspects. Specifically, wearing an opposite-sex VB: induced a modification of subjective feelings of femininity (women felt less feminine) and masculinity (men felt less masculine); balanced the strength of implicit associations between both genders and the self; updated gender-related stereotypes about one's own personality. The studies summarized above provide crucial evidence in support of the hypothesis that wearing an opposite-sex VB may be associated with behavioral changes coherent with the specific features of the embodied avatar (sex, in this case) and the cognitive representations and behaviors that are linked to them.

Vicarious feelings. Vicarious tactile sensations have been reported in healthy people observing pictures (Schirmer et al., 2015) and videos (Morrison et al., 2011; Walker et al., 2017), as well as in people observing representations of their own and others' VBs (Fusaro et al., 2016, 2019, 2021). Our IVR paradigm extends previous knowledge by investigating vicarious feelings elicited by caresses on a same-sex or opposite-sex VB seen from 1PP and thus perceived as one's own. Recent work from our research group shows that it is possible to simulate a virtual environment in which participants experience virtual caresses on several body parts from different virtual characters. The results reflect individuals' common reactions to real-life touch exchanges (Fusaro 
et al., 2021). Here, we expand on these findings by showing how sex-related body swaps can modulate the vicarious feeling of being touched on different regions of one's own VB.

Vicarious reactivity to virtual touches on a virtual body that is sexually congruent or incongruent with the participant's real body. Our present results show that vicarious feelings of touch in heterosexual men were higher when they were caressed by a female avatar. Heterosexual women, on the other hand, did not show any difference in vicarious feelings based on the sex of the touching avatar. Analyses of pleasantness and erogeneity ratings revealed that overall, both sexes preferred cross-sex touch in terms of the feeling of erogeneity elicited by vicarious touch on intimate areas. Additionally, correlation analyses showed that these feelings were stronger when ownership for the same-sex VB was higher. Our results are also coherent with theories suggesting that women engage more frequently in touch-mediated interactions and are more willing to be touched by members of both sexes, even strangers (as in our study), provided that the touch does not have sexual connotations (Stier \& Hall, 1984; Russo et al., 2019; see also SI Appendix, Additional results for results on the appropriateness of virtual caresses). Physiology results parallel the behavioral ones, supporting the notion that the simulation of social interactions in virtual environments may elicit body activation coherent with that experienced during real-life events. Heart rate deceleration (HRD), interpreted as a cardiac function reduction associated with attentional shift contingent upon the processing of an arousing event (Bradley et al., 2001), was found in men for virtual caresses on intimate areas. When asked to rate their feelings of arousal related to the virtual caresses, men considered virtual caress on intimate areas more arousing than caresses on neutral areas (see SI Appendix, Additional results for analyses of the arousal elicited by virtual caresses). Similarly, SCR is considered a measure of sympathetic nervous system involvement related to the processing of arousing stimuli, regardless of their valence (Bradley et al., 2001). While men showed a higher SCR for caresses from a female touching avatar, coherent with the relevance of cross-sex touch in this demographic group, women experienced a higher SCR for both social and intimate touch (compared to neutral touch), paralleling behavioral results on the pleasantness of caresses.

An important result of our study is related to how experiencing an opposite-sex VB in 1PP affects vicarious tactile feelings. For both men and women, we predicted that embodying an opposite-sex VB would shift participants' preferences for touch-mediated interactions towards those of the opposite sex. This is indeed what we found. Heterosexual men rated caresses on intimate body areas from a touching male avatar as more pleasant and more erogenous when they embodied a female body. This change was stronger for the vicarious feeling of erogeneity. Moreover, heterosexual women rated caresses from a female touching avatar in intimate areas as more pleasant and more erogenous when they embodied a male VB. As such, despite the fact that participants in both studies felt greater ownership over a same-sex VB, experiencing an oppositesex VB was found to elicit changes in explicit reactions to same-sex virtual caresses. It is worth noting that, although our results might stem from merely witnessing a preferred (cross-sex) erogenous scenario during the opposite-sex embodiment conditions, correlation analyses support the hypothesis that it was in fact the experience of owning an opposite-sex body that shaped participants' choices. Especially for men, we observed a significant positive correlation between erogeneity and $\mathrm{BO}$ scores for the condition in which they embodied a female body and were virtually touched on intimate areas by a male touching avatar (Fig. 2C). Tellingly, our results indicate that participants' physiological activation differently predicts behavioral outcomes (ratings at VAS scales) and suggest that implicit physiological reactivity may underlie full-body illusions in IVR, discriminate between different virtual scenarios, and predict how people behave in such circumstances. In our case, a decrease in HR and an increase in SCR - two correlates of enhanced processing of arousing stimuli (Bradley et al., 2001) - were associated with increments in erogeneity and pleasantness ratings when heterosexual men and women were caressed by a same-sex toucher and embodied an opposite-sex VB, which further supports the effectiveness of our sex-related body swap illusion. 
Interpersonal touch preferences, especially those concerning intimate touch, are tightly linked to people's SO (Gallace \& Spence, 2010). Our results provide novel insights on the association between $\mathrm{SO}$ and tactile preferences and on the moderation effect that body ownership/perception plays on the latter two. We cannot yet speculate on a specific psychological mechanism being at play during the observed changes in tactile preferences - e.g., a temporary modification of gender identity (Tacikowski et al., 2020). It is highly likely, though, that owning an opposite-sex VB leads to an update in high-order cognitive representations of one's own body, which in turn may temporarily affect gender identity, SO, and, ultimately, interpersonal touch preferences (Maister et al., 2015). Anne Fausto-Sterling has recently put forward a theory of gender, sex, and sexual orientation that is solidly based on the embodied cognition account and can provide further theoretical support for our results (Fausto-Sterling, 2019). According to the author, personal and interpersonal experiences, within and with the body, are inextricably intertwined with the development and the expression of gender, sex, and sexual orientation in the child/adolescent. It follows that a modification of bodily features associated with these psychological representations, feelings, and experiences may have consequences as those we observed in our study.

Our results also suggest that, despite their implicit gender bias, men may be more susceptible to our sex-related body swap illusion (details on across-studies analyses of VAS ratings are provided in SI Appendix, Between-subjects analysis), at least concerning its effect on touch-mediated interaction. While the difference between gender-potency IAT scores speaks in favor of a higher bias in men, no across-sample differences in explicit sexist attitudes were observed. Concerning this apparent difference between men's and women's susceptibility to our sex-related body swap illusion, recent evidence suggests that sex differences in body perception processes may be relevant to understanding some of our results (Aleong \& Paus, 2010; Burke et al., 2019). While heterosexual men seem to be more sensitive to self-related information about the opposite sex's bodies, heterosexual women are particularly sensitive to their own bodies (Burke et al., 2019). Patterns of brain activation in response to information related to one's own body also differ across sexes: Women seem to engage in complex cognitive-emotional processing more often than men, with activation reported in the amygdala and prefrontal areas (Kurosaki et al., 2006). Thus, it may be possible that the higher emotional sensitivity to one's own body demonstrated by womenwhich is likely based on paying greater attention to bodily signals (such as interoceptive signals) could interfere with the illusion of owning an opposite-sex VB or at least weaken the consequences of such body swap. Interestingly, the processing of information related to bodies of the opposite sex has been associated with the deactivation of the right temporoparietal junction (rTPJ) in women, a brain region that has previously been linked to perspective-taking abilities $(51,52)$.

\section{Conclusions and future directions}

By capitalizing on IVR, we were able to investigate how heterosexual men and women reacted at subjective and physiological levels to the experience of wearing a same- or opposite-sex VB and seeing it touched on intimate areas by a male or female avatar. IVR allowed us to both create "impossible" scenarios, i.e., swapping people's sex, and to overcome ethical barriers associated with real intimate touch. While embodying a same-sex VB gave rise to heterosexual-like responses as expected, embodying an opposite-sex VB changed these responses as though the human participants were in the position of their embodied avatar. Both heterosexual men and heterosexual women rated same-sex touch on intimate areas as more pleasant and erogenous when they embodied an opposite-sex VB. Interestingly, this change of perspective had stronger effects in men, who also demonstrated stronger feelings of ownership over an opposite-sex VB. While future work is needed to further qualify this finding, we submit that it may be relevant for the current debate fueled by IVR studies $(26,27)$ on domestic violence and sexual harassment perpetrated by men against women. It may be interesting, for example, to explore whether VRmediated physical transformations may help misogynistic men to take on women's physical and mental perspectives. Finally, our study may have important translational implications by inspiring, for example, VR-based support for transgender people during their surgical and hormonal 
transitions as well as applications in which training, rehabilitation, or simply a promotion of social skills is sought, such as empathy and perspective-taking in people who may lack such skills.

\section{Acknowledgments}

This work was supported by a European Research Council (ERC) Advanced Grant 2017, Embodied Honesty in real world and digital interactions (eHONESTY), to S. M. Aglioti. No conflicts of interest, financial or otherwise, are declared by the authors.

\section{References}

- Aleong R., \& Paus T. (2010). Neural correlates of human body perception. Journal of Cognitive Neuroscience, 22(3), 482-495.

- Banakou D., Groten R., \& Slater M. (2013). Illusory ownership of a virtual child body causes overestimation of object sizes and implicit attitude changes. Proceedings of the National Academy of Sciences (PNAS) USA, 110(31), 12846-12851.

- Barton K. (2020). MuMIn: multi-model inference. R package version 1.43.17. https://CRAN.R-project.org/package=MuMIn.

- Bates D., Sarkar D., Bates M. D., Matrix L. (2007). The Ime4 package. R package version, 2(1), 74.

- Berlucchi G., \& Aglioti S. M. (2010). The body in the brain revisited. Experimental Brain Research, 200(1), 25.

- Blanke O., Landis T., Spinelli L., \& Seeck M. (2004). Out-of-body experience and autoscopy of neurological origin. Brain, 127(2), 243-258.

- Botvinick M., \& Cohen J. (1998). Rubber hands "feel" touch that eyes see. Nature, 391(6669), 756-756.

- Bradley M. M., Codispoti M., Cuthbert B. N., \& Lang P. J. (2001). Emotion and motivation I: defensive and appetitive reactions in picture processing. Emotion, 1(3), 276.

- Burke, S. M., Majid, D. A., Manzouri, A. H., Moody, T., Feusner, J. D., \& Savic, I. (2019). Sex differences in own and other body perception. Human Brain Mapping, 40(2), 474-488.

- Carey, M., Crucianelli, L., Preston, C., \& Fotopoulou, A. (2019). The effect of visual capture towards subjective embodiment within the full body illusion. Scientific Reports, 9(1), 1-12.

- de Borst A. W., Sanchez-Vives M. V., Slater M., \& de Gelder B. (2020). First-Person Virtual Embodiment Modulates the Cortical Network that Encodes the Bodily Self and Its Surrounding Space during the Experience of Domestic Violence. eNeuro, 7(3).

- Decety J., \& Lamm C. (2007). The role of the right temporoparietal junction in social interaction: how low-level computational processes contribute to meta-cognition. Neuroscientist, 13(6), 580-593.

- Fusaro M., Tieri G., \& Aglioti S. M. (2016). Seeing pain and pleasure on self and others: behavioral and psychophysiological reactivity in immersive virtual reality. Journal of Neurophysiology, 116(6), 2656-2662.

- Fusaro M., Tieri G., \& Aglioti S. M. (2019). Influence of cognitive stance and physical perspective on subjective and autonomic reactivity to observed pain and pleasure: an immersive virtual reality study. Consciousness and Cognition, 67, 86-97.

- $\quad$ Fusaro, M., Lisi, M. P., Tieri, G., \& Aglioti, S. M. (2021). Heterosexual, gay, and lesbian people's reactivity to virtual caresses on their embodied avatars' taboo zones. Scientific Reports, 11(1), 1-12.Fusco G., Tieri G., \& Aglioti S. M. (2020). Visual feedback from a virtual body modulates motor illusion induced by tendon vibration. Psychological Research, 1-13.

- Gallace A., \& Spence C. (2010). The science of interpersonal touch: an overview. Neuroscience and Biobehavioral Reviews, 34(2), 246-259. 
- Glick P., \& Fiske S. T. (1996). The ambivalent sexism inventory: differentiating hostile and benevolent sexism. Journal of Personality and Social Psychology, 70(3), 491.

- Greenwald A. G., McGhee D. E., \& Schwartz J. L. (1998). Measuring individual differences in implicit cognition: the implicit association test. Journal of Personality and Social Psychology, 74(6), 1464.

- Keenaghan, S., Bowles, L., Crawfurd, G., Thurlbeck, S., Kentridge, R. W., \& Cowie, D. (2020). My body until proven otherwise: Exploring the time course of the full body illusion. Consciousness and Cognition, 78, 102882.

- Kilteni, K., Normand, J. M., Sanchez-Vives, M. V., \& Slater, M. (2012). Extending body space in immersive virtual reality: a very long arm illusion. PloS One, 7(7), e40867.

- Kilteni K., Maselli A., Kording K. P., \& Slater M. (2015). Over my fake body: body ownership illusions for studying the multisensory basis of own-body perception. Frontiers in Human Neuroscience, 9, 141.

- Kinsey A. C., Pomeroy W. R., \& Martin C. E. (2003). Sexual behavior in the human male. American Journal of Public Health, 93 (6), 894-898.

- Kurosaki M., Shirao N., Yamashita H., Okamoto Y., \& Yamawaki S. (2006). Distorted images of one's own body activates the prefrontal cortex and limbic/paralimbic system in young women: a functional magnetic resonance imaging study. Biological Psychiatry, 59(4), 380-386.

- Lenth R. V. (2017). Using Ismeans. Journal of Statistical Software, 69, 1-33.

- Lenth R. V. (2020). emmeans: Estimated Marginal Means, aka Least-Squares Means. R package version 1.5.2-1. https://CRAN.R-project.org/package=emmeans.

- Löken L. S., Wessberg J., McGlone F., \& Olausson H. (2009). Coding of pleasant touch by unmyelinated afferents in humans. Nature Neuroscience, 12(5), 547.

- Maister, L., Slater, M., Sanchez-Vives, M. V., \& Tsakiris, M. (2015). Changing bodies changes minds: owning another body affects social cognition. Trends in Cognitive Sciences, 19(1), 6-12.

- Manganelli Rattazzi A. M., Volpato C., \& Canova L. (2008). L'atteggiamento ambivalente verso donne e uomini. Un contributo alla validazione delle scale ASI e AMI. Giornale Italiano di Psicologia, 35(1), 217-246.

- Monti A., \& Aglioti S. M. (2018) Flesh and bone digital sociality: On how humans may go virtual. British Journal of Psychology, 109(3), 418-420.

- Monti A., Porciello G., Tieri G., \& Aglioti S. M. (2020). The "embreathment" illusion highlights the role of breathing in corporeal awareness. Journal of Neurophysiology, 123(1), 420-427.

- Moro, V., Pernigo, S., Tsakiris, M., Avesani, R., Edelstyn, N. M., Jenkinson, P. M., \& Fotopoulou, A. (2016). Motor versus body awareness: voxel-based lesion analysis in anosognosia for hemiplegia and somatoparaphrenia following right hemisphere stroke. Cortex, 83, 62-77.

- Morrison I., Björnsdotter M., \& Olausson, H. (2011). Vicarious responses to social touch in posterior insular cortex are tuned to pleasant caressing speeds. Journal of Neuroscience, 31(26), 9554-9562.

- Morrison I., Löken L. S., \& Olausson H. (2010). The skin as a social organ. Experimental Brain Research, 204(3), 305-314.

- Neyret, S., Navarro, X., Beacco, A., Oliva, R., Bourdin, P., Valenzuela, J., ... \& Slater, M. (2020). An embodied perspective as a victim of sexual harassment in virtual reality reduces action conformity in a later Milgram obedience scenario. Scientific Reports, 10(1), 1-18.

- $\quad$ Pavone, E. F., Tieri, G., Rizza, G., Tidoni, E., Grisoni, L., \& Aglioti, S. M. (2016). Embodying others in immersive virtual reality: electro-cortical signatures of monitoring the errors in the actions of an avatar seen from a first-person perspective. Journal of Neuroscience, 36(2), 268-279. 
- Peck T. C., Doan M., Bourne K. A., \& Good J. J. (2018). The effect of gender body-swap illusions on working memory and stereotype threat. IEEE Transactions on Visualization and Computer Graphics, 24(4), 1604-1612.

- $\quad$ Peck T. C., Good J. J., \& Bourne K. A. (2020). Inducing and mitigating stereotype threat through gendered virtual body-swap illusions. Proceedings of the $2020 \mathrm{CHI}$ Conference on Human Factors in Computing Systems, Honolulu, HI, USA.

- Peck T. C., Seinfeld S., Aglioti S. M., \& Slater M. (2013). Putting yourself in the skin of a black avatar reduces implicit racial bias. Consciousness and Cognition, 22(3), 779-787.

- Petkova V. I., \& Ehrsson H. H. (2008). If I were you: perceptual illusion of body swapping. PloS One, 3(12), e3832.

- Preston, C., \& Ehrsson, H. H. (2014). Illusory changes in body size modulate body satisfaction in a way that is related to non-clinical eating disorder psychopathology. PloS One, 9(1), e85773.

- Provenzano, L., Porciello, G., Ciccarone, S., Lenggenhager, B., Tieri, G., Marucci, M., ... \& Bufalari, I. (2020). Characterizing body image distortion and bodily self-plasticity in anorexia nervosa via visuo-tactile stimulation in virtual reality. Journal of Clinical Medicine, 9(1), 98.

- Pyasik, M., Tieri, G., \& Pia, L. (2020). Visual appearance of the virtual hand affects embodiment in the virtual hand illusion. Scientific Reports, 10(1), 1-11.

- $\quad$ R Core Team (2019). R: a language and environment for statistical computing. $R$ Foundation for Statistical Computing, Vienna, Austria. https://www.R-project.org/.

- Rudman L. A., Greenwald A. G., \& McGhee D. E. (2001). Implicit self-concept and evaluative implicit gender stereotypes: self and ingroup share desirable traits. Personality and Social Psychology Bulletin, 27(9), 1164-1178.

- Russo V., Ottaviani C., \& Spitoni G. F. (2019). Affective touch: a meta-analysis on sex differences. Neuroscience and Biobehavioral Reviews, 108, 445-452.

- Sanchez-Vives M. V., Spanlang B., Frisoli A., Bergamasco M., \& Slater M. (2010). Virtual hand illusion induced by visuomotor correlations. PLoS One, 5(4), e10381.

- Schirmer, A., Reece, C., Zhao, C., Ng, E., Wu, E., \& Yen, S. C. (2015). Reach out to one and you reach out to many: Social touch affects third-party observers. British Journal of Psychology, 106(1), 107-132.

- Seinfeld, S., Arroyo-Palacios, J., Iruretagoyena, G., Hortensius, R., Zapata, L. E., Borland, D., ... \& Sanchez-Vives, M. V. (2018). Offenders become the victim in virtual reality: impact of changing perspective in domestic violence. Scientific Reports, 8(1), 1-11.S.

- Slater M., \& Sanchez-Vives M. V. (2014). Transcending the self in immersive virtual reality. Computer, 47(7), 24-30.

- Slater M., Spanlang B., Sanchez-Vives M. V., Blanke O. (2010). First person experience of body transfer in virtual reality. PloS One, 5(5).

- Stier D. S., \& Hall J. A. (1984). Gender differences in touch: an empirical and theoretical review. Journal of Personality and Social Psychology, 47(2), 440.

- Tacikowski P., Fust J., Ehrsson H. H. (2020). Fluidity of gender identity induced by illusory body-sex change. Scientific Reports, 10, 14385.

- Vallar G., \& Ronchi R. (2009). Somatoparaphrenia: a body delusion. A review of the neuropsychological literature. Experimental Brain Research, 192(3), 533-551.

- Van Der Hoort, B., Guterstam, A., \& Ehrsson, H. H. (2011). Being Barbie: the size of one's own body determines the perceived size of the world. PloS One, 6(5), e20195.

- Walker S. C., Trotter P. D., Woods A., \& McGlone F. (2017). Vicarious ratings of social touch reflect the anatomical distribution \& velocity tuning of $\mathrm{C}$-tactile afferents: a hedonic homunculus? Behavioral Brain Research, 320, 91-96.

- Wang H., Callaghan E., Gooding-Williams G., McAllister C., \& Kessler K. (2016). Rhythm makes the world go round: an MEG-TMS study on the role of right TPJ theta oscillations in embodied perspective taking. Cortex, 75, 68-81 (2016). 